\title{
El diario de campo como estrategia educativa/reflexiva en la práctica quirúrgica de enfermería
}

\section{The diary of field as educational/reflective strategy in practice surgical nursing}

\author{
Claudia Patricia Bonilla Ibáñez 1 , Madeleine Cecilia Olivella Fernández ${ }^{1}$ y Clara Victoria Bastidas \\ Sánchez 1 \\ ${ }^{1}$ Universidad de Tolima. Ibagué, Colombia \\ *Dirigir correspondencia a: cbonilla@ut.edu.co
}

\section{Proceso Editorial \\ Recibido: 260520 \\ Aceptado: 260121 \\ Publicado: 230221}

DOI 10.17081/innosa.115

(C)Copyright 2021.

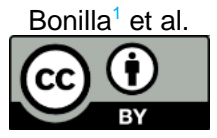

\section{RESUMEN}

Introducción: El diario de campo o diario reflexivo, es una herramienta útil en la valoración del aprendizaje de los estudiantes en áreas de la salud, no solamente de los procesos o procedimientos realizados a las personas enfermas, sino de aquellos aspectos integrales para la formación personal y profesional. Métodos: Investigación cualitativa, descriptiva, de análisis de contenido realizado entre 2018 y 2019 con 54 estudiantes de VII semestre del programa de enfermería en el servicio de cirugía de un Hospital de tercer nivel en la ciudad de lbagué, a los cuales se les entrego un formato de diario de campo para diligenciar cada día de acuerdo a la asignación diaria. La información se analizó en software ATLAS Ti versión 6. Resultados: Los resultados se presentan en una red semántica que comprende 5 categorías emergentes así: adquisición de habilidades y reforzamiento de conceptos, concepto del campo de práctica, autocorrección, reflexiones del cuidado, actitudes del docente y personal de salud. Conclusiones: Los estudiantes consideran que el diario reflexivo es una herramienta importante para valorar la práctica diaria y sugieren se utilice en otros espacios de aprendizaje, pueden identificar y describir los aspectos relacionados no solo con los procedimientos sino con aspectos relacionados con el campo de práctica, el acompañamiento docente, el apoyo del personal del servicio, entre otros.

Palabras clave: Diario de campo; cirugía; aprendizaje; docente de enfermería.

\section{ABSTRACT}

Background: The field diary is a tool for the evaluation of student learning in areas of health, not only of the processes or procedures performed on patients but those integral aspects for personal training. Methods: Descriptive, qualitative study was conducted between 2018 and 2019 with 54 students of VII semester nursing program in the surgery department of a tertiary hospital in the city of lbague, to whom he handed a format field diary to fill each day according to the daily allowance. The information was analyzed in ATLAS Ti software version 6 . Results: the data were classified in a semantic network with 5 categories: Acquisition of skills and reinforcement of concepts, concept field practice, AutoCorrect, reflections of care, attitudes of teachers and health workers. Conclusions: Students believe that this tool is important to assess their practice and suggest it be used in other learning spaces can identify and describe the related aspects with not only the procedures but with aspects related to the practice field, accompaniment teachers, support staff service, among others.

Keywords: Diary of fields; surgery; learning; teaching nursing. 


\section{INTRODUCCIÓN}

En la formación de profesionales de ciencias de la salud es esencial el desarrollo de una práctica reflexiva especialmente en el ámbito clínico. El escenario real de las instituciones de salud, puede ser impredecible y estresante para muchos estudiantes, particularmente durante el período inicial de estas prácticas (1). El profesor o tutor clínico requiere supervisar y acompañar el proceso formativo de los estudiantes en forma permanente, teniendo en cuenta la responsabilidad ética frente a la vida de las personas, sujetos de cuidado. Por lo tanto, requiere diariamente de herramientas útiles que evalúen tanto el proceso de aprendizaje como la práctica propia del quehacer de la profesión de enfermería.

El diario de campo o diario reflexivo consiste en un documento que genera el estudiante durante sus periodos de prácticas clínicas en el que plasma sus actividades problemas, sentimientos y todo aquello que éste considera relevante, siendo éste el instrumento elegido para estimular la reflexión de los estudiantes durante sus periodos de prácticas clínicas. Según Medina, su escritura fomenta un proceso de reflexión a través del cual los estudiantes acceden al conocimiento profesional de manera profunda, comprensiva y reflexiva, opuesta al aprendizaje memorístico potenciando las habilidades de pensamiento y permitiendo el desarrollo de comprensiones profundas como procesos de construcción de significados (2).

El diario de campo constituye una herramienta formativa que promueve la reflexividad y la constitución de eticidad, por el valor hermenéutico que contiene en sí mismo como facilitador de la comprensión de la complejidad del ámbito clínico, al dotar de significado cada una de las experiencias que el estudiante afronta. Es considerado como una herramienta de gran utilidad para los maestros, no sólo como posibilidad de escritura ni como narración anecdótica de lo que sucede en la clase o escenarios de práctica, su finalidad es formativa y es elemento clave para la investigación.

El diario de campo ha sido un método de las ciencias sociales que permite narrar en forma sincrónica (3) lo que ocurre dando sentido a la realidad. En enfermería robustece el conocimiento disciplinar y profesional al contribuir al desarrollo del patrón de conocimiento personal que expresa el conocimiento de sí mismo (en relación con los demás); desde la perspectiva intrínseca se considera un patrón fundamental, se requiere el desarrollo del conocimiento personal; porque es el más problemático para dominar y enseñar, pero a la vez es esencial para entender el significado de la salud (4).

Existe consenso en que las prácticas constituyen una oportunidad en la que profesores en formación transfieren los conocimientos teóricos aprendidos hacia escenarios y contextos educativos reales, lo que permite un aprendizaje experiencial que favorece el desarrollo de un pensamiento reflexivo respecto del propio ejercicio de la profesión, en el caso de enfermería y en su formación, la teoría y la práctica se complementan con la finalidad de acercar al estudiante con la realidad asistencial que le permita lograr un aprendizaje significativo (ㅁ).

La práctica implica el aprender haciendo de las/los estudiantes a través de la exposición y la inmersión, las conversaciones y trabajo colaborativo con las enfermeras tutoras y compañeras, la adquisición de un arte cuasi autónomo de la práctica clínica, el vínculo transaccional entre la enseñanza del aula y los aprendizajes experienciales de la práctica (ㅁ). 
El diario de campo es una estrategia que favorece la humanización del cuidado, a partir del ejercicio narrativo-reflexivo que el suscita, tanto el profesor como el estudiante se ven abocados a una experiencia de aprendizaje significativo que promueve un acercamiento al ser humano permitiéndole compartir experiencias y sentimientos que conduce a abordajes de cuidado individualizados, dando respuestas a sus necesidades (7). Este ejercicio es particularmente valioso en escenarios como la práctica clínica en áreas quirúrgicas, algunos estudios describen el poco apoyo que se les da a los estudiantes en los quirófanos: se sienten menos apoyados por los profesionales, y al contrario de otras unidades, experimentan miedo, inseguridad, desconocimiento, incomodad, aislamiento, estorbo y dificultad en un espacio que es desconocido para ellos (ㅇ).

Este estudio se justifica ante la necesidad de implementar nuevas formas de evaluación en el proceso formativo. Se utiliza el diario de campo como una herramienta que permite sistematizar las experiencias para luego analizar los resultados. De hecho, se puede utilizar desde el mismo inicio de un estudio, tomando notas de manera organizativa: fechas y lugar de encuentros, nombre de actividades e involucrados, descripción, reflexiones, ( $\underline{9})$ entre otros aspectos que se consideren relevantes. Este estudio de forma preliminar pretende describir reflexiones de los estudiantes de enfermería durante sus prácticas clínicas en el área de salas de cirugía en un hospital de tercer nivel de atención, teniendo en cuenta aspectos relacionados con: rol de desempeño, aprendizaje realizado con anterioridad, reconocimiento de aprendizajes para reforzar, y aspectos positivos y negativos relacionados con el docente.

\section{MÉTODOS}

Se realiza un estudio descriptivo, de abordaje cualitativo a partir del análisis de contenido de los diarios de campo. Esta metodología es congruente con lo enunciado por Piñuel (2002), Tinto Arandes (2013) Arbeláez \& Onrubia (2014) Díaz Herrera, (2018), (10) indican el uso del análisis de contenido en investigaciones descriptivas que sirven de punto de partida para posteriores investigaciones, la técnica del análisis de contenido debido a sus características constituye una importante herramienta metodológica para la realización de una investigación descriptiva (11).

El proyecto se realizó con estudiantes de la Facultad de Ciencias de la Salud de la Universidad del Tolima del programa de enfermería, en el curso de enfermería del adulto, cátedra cirugía séptimo semestre, dentro de la cual realizan una rotación en salas de cirugía, de 6 días con una duración de 6 horas los martes, miércoles y jueves en la jornada de la mañana durante los años 2018 -2019. La unidad de análisis fueron un total de 198 diarios de campo, se diligenciaron por parte de los estudiantes, de acuerdo con la asignación de actividades como circulante de sala, circulante de anestesia, instrumentación y atención a pacientes en la unidad de cuidado posanestésico.

El formato utilizado fue físico, teniendo en cuenta que tendría al inicio día, mes, año, horario y asignación diaria, luego se dividió cada hoja en dos partes o columnas, anotando los datos procedentes de la observación en el lado izquierdo y la reflexión o comentario sobre dichas observaciones en el lado derecho. Al final se incluyó la firma del estudiante que elaboro el diario de campo (consentimiento informado) esto de acuerdo con la estructura para este tipo de documentos (12). 
Los datos recogidos se digitaron y se consolidaron en el software para análisis cualitativo ATLAS Ti versión 6.

El análisis de la información se realizó teniendo en cuenta la técnica de análisis de contenido de los diarios de campo realizados por los estudiantes se determinaron las siguientes fases: Delimitación de objetivos del estudio, selección de la unidad de análisis, preparación de las unidades de análisis para el procesamiento, Recuento a través de software Atlas ti, delimitación de descriptores emergentes, categorización de códigos emergentes. Se tomaron la totalidad de los diarios realizados, teniendo en cuenta que estuvieran diligenciados en su totalidad de acuerdo con la guía entregada a los estudiantes previamente y que hubieran sido revisados por la docente.

Los aspectos éticos de la investigación, se enmarcan en la política de protección de datos del país, los registros de diario de campo se anonimizaron con códigos, su uso es solamente con fines académicos y de investigación, para esto se solicitó firmaran en cada diario elaborado el respectivo documento de consentimiento, posterior a la comprensión del proceso para la utilización de los mismos con fines de investigación. Se explicó a los estudiantes que adicional al propósito evaluativo de la rotación los diarios se utilizarían con fines investigativos para validar inicialmente el uso didáctico de la herramienta y posteriores investigaciones que puedan abordar nuevos desarrollos e llevó a cabo un estudio observacional, de corte transversal, durante el período comprendido entre el 15 de enero y 15 de marzo del 2020, mediante la aplicación de encuestas de un instrumento utilizado y autorizado por Hincapie y Col (ㅁ), a mujeres universitarias entre 17 y 30 años de edad, que pertenecieran a la facultad de ciencias de la salud de la Universidad Pedagógica y Tecnológica de Colombia y fueran usuarias de métodos de anticoncepción hormonales en el presente o el pasado; se incluyeron a quienes cumplieran dichos criterios y a la vez aceptaran el debido consentimiento informado para el uso de la información.

Se calculó el tamaño de la muestra con el software Epiinfo versión 7,2, tomando como base la población total de mujeres registradas en el sistema de admisiones y registro de la Universidad que cumplieran los criterios de selección, incluyendo a mujeres entre los 17 a 30 años de edad, usuarias de métodos de anticoncepción, pertenecientes a alguna de las dos facultades de ciencias de la salud mencionadas, excluyendo aquellas que diligenciaran la encuesta de forma incorrecta, incompleta o que no aceptaran la participación en el estudio. El número total de la población accesible se calculó a través de la fórmula: número total de mujeres registradas $\mathrm{x}$ proporción que se espera usen anticoncepción hormonal (30\%). La población accesible basados en los datos de mujeres inscritas en los programas de la facultad de ciencias de la salud dio un total de 1500 mujeres, para el tamaño de la muestra de estimó una proporción de abandono del método anticonceptivo de $20 \%$ y nivel de confianza de $95 \%$, dando un total de 580 mujeres a encuestar.

Se realizó muestreo por conveniencia. El muestreo no se realizó para obtener información estadística sobre el fallo terapéutico, pero sí sobre los abandonos de la terapia, así como para evaluar descriptivamente las posibles causas de fallo.

Las variables estudiadas correspondían a datos sociodemográficos, no adherencia (interrupción del uso de anticoncepción hormonal por más de 30 días), suspensión del método 
de planificación (modificación de la sustancia de anticoncepción) y eficacia terapéutica (embarazo no deseado).

Análisis estadístico: Mediante el uso de los programas de Excel 2007 (para la creación de una base de datos) y SPSS Stadistics 22, se realizó un análisis estadístico descriptivo univariado, y adicionalmente, se analizó independientemente el subgrupo de casos en los que se presentó fallo terapéutico.

Sesgos: Se pueden encontrar sesgos de selección e información, puesto que las participantes en algunos casos pudieron suministrar información que no era concordante con la realidad, además del sesgo de memoria que se podía presenta; sin embargo, para controlar estos sesgos, se hizo una prueba piloto para evaluar la redacción y facilidad de entendimiento de la encuesta, con lo cual se detectaron algunos errores y se modificaron, adicionalmente se dieron instrucciones claras para el diligenciamiento de los cuestionarios aplicados.

Consideraciones éticas: De acuerdo con la resolución 8430 de 1993, mediante la cual se dictan los criterios de investigación en salud, se establece que esta es una investigación sin riesgo. Además, a cada participante se le dio el debido consentimiento informado para el uso de la información suministrada.

\section{RESULTADOS}

En este estudio participaron 54 estudiantes, 21 hombres y 33 mujeres. De los 198 diarios revisados 75 los realizaron hombres y 123 mujeres. La distribución por asignación fue así: Circulante de sala: 50; Circulante de anestesia: 47; rol en instrumentación: 51; Unidad de cuidado post anestésico: 50 diarios de campo elaborados.

En la Figura 1 se muestra la representación gráfica de la categorización de las afirmaciones realizadas por los participantes, referido al nodo central: Diario de campo de los roles de los estudiantes en sala de cirugía. Ahora bien, la información registrada en los diarios de campo por los estudiantes está distribuida en los diferentes roles en el quirófano: Circulante de Sala y Anestesia, rol de Instrumentador y recuperación; una vez analizadas y agrupadas dio origen a seis categorías fundamentales llamadas: Adquisición de habilidades, Reforzamiento de conceptos, Actitudes del docente y personal de la institución, Concepto del campo de práctica, Autocorrecciones y por último Reflexiones del cuidado.

De acuerdo con la primera categoría, nombrada adquisición de habilidades se establece el aprendizaje de los estudiantes de forma positiva y relevante al emplear, aplicar e identificar los conocimientos y habilidades adquiridos en niveles teóricos, en las prácticas quirúrgicas, constituyendo o formando el saber y saber hacer de sus actividades. En los niveles prácticos académicos los estudiantes consideran que también modifican sus habilidades al trabajar en los escenarios de cuidados, lo cual representa un aspecto relevante en el área de enfermería, por la necesidad de acortar la brecha teórica-practica de su labor profesional. Asimismo, los estudiantes describieron en esta categoría "satisfacción por realizar actividades nuevas", "conocimientos y concentración para realizar las actividades", por ende, reconocen que la repetición de prácticas fomenta sus habilidades al poseer iniciativa y oportunidad de realizar procedimientos quirúrgicos, por lo cual enfatiza el servicio como un ambiente agradable, sugiriendo permanecer más días para el complemento de sus conocimientos. 


\section{Figura 1. Red semántica de los diarios de campo}

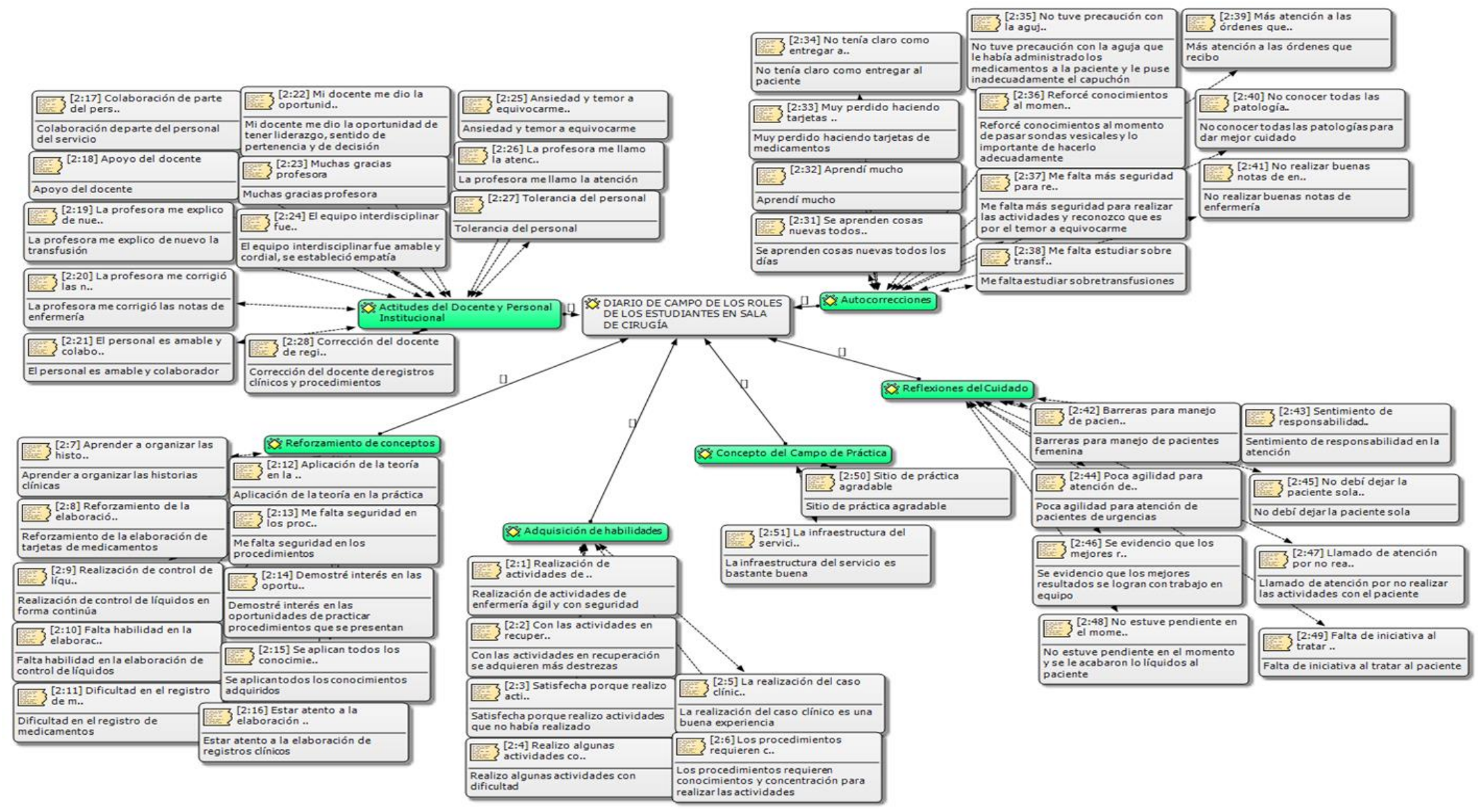

Fuente: Elaboración propia 
Por su parte, el reforzamiento de concepto, no es más que repasar las informaciones teóricas previas ya obtenidas para el complemento de sus conocimientos o aprendizaje ante las prácticas con el objetivo de alcanzar un aprendizaje significativo, al reconstruir ambas informaciones en una definición propia, favoreciendo un pensamiento crítico y reflexivo propio del ejercicio de la profesión. Comprende los datos e informaciones importantes para el desarrollo eficaz de las prácticas quirúrgicas, sin embargo, los estudiantes enfatizan inseguridad y reflexión en el hecho de que los procedimientos de enfermería requieren de reforzamiento de los conocimientos en cuanto a la "aplicación de la teoría en la práctica", "seguridad en los procedimientos", así como también "reforzamiento en la elaboración de tarjetas de medicamentos y control de líquidos" (Ver Figura 1). Por ello, toda actividad realizada en las áreas pautadas requiere de seguridad y atención de las informaciones teóricas previas obtenidas como el de su labor personal individual para el complemento de las actividades de circulantes de sala y anestesia, rol de Instrumentador y recuperación.

Además, la categoría de actitudes del docente y personal de la institución, es definida como el comportamiento tanto del docente como el personal médico ante las actividades prácticas realizadas por los estudiantes en las distintas áreas, en ella tanto los docentes como el personal pueden demostrar sus estados de ánimo como: felicidad, desilusión o enojo ante los avances académicos evidenciados. Cabe agregar, que dicha categoría complementa y fundamenta el aprendizaje de los estudiantes para su formación como ciudadano profesional. En efecto, muchos estudiantes visibilizan aspectos como "Corrección de los registros clínicos y de los procedimientos a llevar a cabo", "apoyo del docente" "oportunidad de liderazgo" "tolerancia del personal" y adicionalmente "la colaboración por parte del personal de servicio". No obstante, aunque se encuentra ansiedad y temor de equivocación, la actitud del personal siempre hace parte del aprendizaje. Por esta razón, es importante e incluso innovador el trabajo y apoyo del equipo de salud en cada una de las asignaciones, ya que permiten reforzar y fortalecer los conocimientos teóricos-prácticos y el vínculo con el trabajo que se realizara al finalizar los estudios, por ende, siempre se valora la necesidad de buenas relaciones interpersonales.

El concepto del campo de práctica es otra de las categorías establecida registrada en los diarios de campo, la cual permite llevar a cabo actividades prácticas en la institución hospitalaria, donde los estudiantes tienen la oportunidad de ejercer y ampliar sus conocimientos prácticos de acuerdo con la teoría aprendida. En las prácticas clínicas quirúrgicas llevadas a cabo en los distintos escenarios establecidos, los estudiantes en el servicio de sala manifiestan ansiedad y estrés por la agilidad con la que es realizado los procedimientos y también motivación por la experiencia enriquecedora brindada por prácticas productivas e interesantes, establecen que las "infraestructuras del servicio en los sitios de prácticas es agradable, organizado y bastante buena". Al establecer como prioridad la importancia de un campo adecuado y tolerante para la permanencia de los estudiantes permite abiertamente la retroalimentación de procesos tanto académicos como asistenciales.

En cuanto a la categoría de autocorrecciones, se pude decir que es la forma donde se corrigen los errores cometidos en las prácticas quirúrgicas realizada por los estudiantes, en otras palabras, la autocorrección es la capacidad o habilidad empleada por los docentes y el personal de la institución para mejorar y mediar los errores cometidos por los estudiantes con los objetivos de que estos reflexionen para obtener un aprendizaje significativo de ello. En esta 
categoría los estudiantes indican la forma en cómo realizan las autocorrecciones y reflexiones sobre los hechos realizados, enfatizan que todos lo días se aprenden nuevas informaciones; muchas de manifestaciones realizada por los estudiantes en esta categoría son "me hace dar cuenta de la responsabilidad que se debe tener ante los pacientes" lo cual es un comentario imprescindibles ya que en toda practica quirúrgica del personal de enfermería se requiere de atención, control y seguridad, puesto a que muchas de sus manifestación son "soy consciente que debo mejorar", "me sentí un poco pérdida", debo "poner más atención a las órdenes que recibo", "me falta más seguridad para realizar las actividades y reconozco que es por el temor a equivocarme", "no tenía claro como entregar al paciente"; igualmente establecen que al estar con pacientes delicados les resulta "traumático y entristecedor". Por ende, el docente en conjunto con en el equipo de salud juegan un rol importante como formadores de las habilidades y destrezas del enfermero quirúrgico, por dicha razón son reconocidos de forma importante ya que su aprendizaje es considerando reflexivo y eficiente tanto por parte de los estudiantes como del equipo de salud.

Finalmente, la última categoría llamada reflexión del cuidado, es un aspecto importante a desarrollar en los estudiantes, ya que esta facilita el análisis de los cuidados y procedimientos a emplear en los diferentes pacientes, a partir de esta habilidad reflexiva todo estudiante tienen la capacidad de realizar su mirada a partir de su propio quehacer, favoreciendo su construcción personal o bien, de sí mismo como un sujeto ético, responsable y comprometido ante las actividades quirúrgicas. Igualmente, el estudiante establece que el "el tiempo de práctica es corto" evidenciado su interés y motivación en aprender, además enfatizan manifestaciones como "barreras para el manejo de pacientes femeninas", "falta de iniciativa y ganas de aprender", "considero que hay que vivir ciertas experiencias para hacernos más fuertes", "no estuve pendiente en el momento y se le acabaron los líquidos", los cuales representan el momento para llevar a cabo una reflexión y buscar mejoras en su labor. Cabe mencionar que tanto el estudiante como docente reconocen como vital importancia el tiempo, el cual nunca es suficiente para permanecer con aquellos pacientes que requieren de constante dedicación para poder brindar un cuidad de calidad acorde a sus necesidades.

\section{DISCUSIÓN}

Este estudio fortalece la información sobre la utilidad del diario de campo o diario reflexivo en enfermería. Constituye un aporte específico en el desarrollo e implementación de estrategias pedagógicas para el fortalecimiento del aprendizaje de los estudiantes en el área quirúrgica que es un campo nuevo y fructífero en la exploración. El diario de campo debe abordarse con un enfoque reflexivo, y motivador para la construcción de elementos fundamentales en la formación integral del estudiante y del docente, permite explorar procesos de la práctica que ayudan a que esta sea productiva, agradable y que brinde los elementos necesarios para el fortalecimiento del enfermero quirúrgico.

En el área quirúrgica para este estudio, el diario de campo se consolidó con las concepciones tanto iniciales como finales del estudiante incluyendo las interacciones con compañeros, docente y personal de salud. Estas construcciones tienen elementos diversos por cuanto los micro escenarios varían de acuerdo a los objetivos a alcanzar por parte del estudiante; sin embargo y teniendo en cuenta que se desempeña como circulante de sala, de anestesia, rol de instrumentadora y enfermera(o) en recuperación, el estudiante integra los elementos antes 
mencionados para adquirir habilidades, destrezas y aprendizajes que cumplan con los objetivos propuestos para la práctica y contribuyan a su desempeño profesional. La retroalimentación y reflexión grupal e individual con el docente, compañeros y equipo de salud fortalecerá las bases teóricas y prácticas del futuro enfermero.

En las categorías emergentes la de adquisición de habilidades y reforzamiento de conceptos: En este punto, el trabajo de los estudiantes de enfermería, apoyados con el diario de campo, se constituiría en una herramienta con la finalidad de consolidar la formación de pregrado, la cual se ha podido demostrar, en otras investigaciones, que es insuficiente para llevar el liderazgo profesional, lo cual solo es posible en el campo de práctica, los enfermeros como líderes deben ser capaces de influenciar a sus trabajadores de manera positiva para lograr alcanzar resultados y beneficios deseados, para esto es necesario que adquieran las competencias necesarias $(\underline{13}, \underline{14})$.

Asimismo, los estudiantes han señalado que el trabajo en la práctica les hace reflexionar sobre los mecanismos para reforzar el conocimiento, considerando el diario de campo, como un procedimiento para hacerlo. Este hallazgo es coherente con el hecho relacionado con el desarrollo de habilidades y competencias determinadas, debido al uso del diario de campo como una vía para el desarrollo de mayores habilidades y la búsqueda de resultados satisfactorios (15). En consecuencia, las habilidades se desarrollan con la capacitación, la búsqueda personal, el quehacer docente, el desempeño profesional y la motivación intrínseca del estudiante.

Sobre esto último, la motivación de un estudiante es producto de su percepción sobre el avance en experiencias y aprendizaje, lo cual es logrado en la práctica y la sistematización constante con el diario de campo (16). Esto le genera mayor confianza para el abordaje de retos personales y pensar más allá de conseguir una nota, por el contrario, llenarse de satisfacción por el aprendizaje de habilidades nuevas y la resolución de desafíos.

Sumado a esto se profundiza en el constructo de las actitudes del docente y personal de la institución, en donde los estudiantes han señalado la importancia de la retroalimentación y el aprendizaje durante su práctica. En este sentido se resalta que el estudiante cuando está presto a aprender de una experiencia, puede construir el aprendizaje durante su trayectoria, en este caso la práctica (15). Ahora bien, asimismo sucede con los profesores, quienes deben estar prestos a desempeñar un doble papel, de aprendiz y de educador, tomando las nuevas experiencias de los diarios de campo y aprender de ellas, como el de tomar sus experiencias propias y enriquecer el quehacer del estudiante de enfermería.

También se ha recolectado, desde los diarios de campo, percepciones sobre la orientación y colaboración por parte del personal de la institución como por parte del profesor. Esto ayuda en gran medida, en el sentido de la utilidad del apoyo para corregir posibles actos con enlaces desfavorables, creando una mayor conciencia y siendo punto de partida para continuar mejorando la práctica del estudiante (17). Entonces, el estudiante puede mejorar a diario su sentimiento de presión o posible ansiedad frente a la posibilidad de cometer un error, esto, basado en los posibles comentarios y retroalimentaciones dadas por los profesores en un diario de campo, considerado por otras investigaciones, como una herramienta de gran aporte 
al aprendizaje por feedback (17). Asimismo, la retroalimentación debería hacerse lo más rápido posible, durante la pasantía o posteriormente al procedimiento efectuado por el practicante (17).

De otro lado, sobre el constructo del concepto de campo de práctica, los estudiantes lo ven como una tarea enriquecedora, siendo producto de un proceso, en donde se adaptan con el tiempo, así como lo señalan otras investigaciones, los estudiantes se sienten inseguros durante las primeras semanas y van ganando confianza con el paso de las mismas (18). Quizá este avance y evolución es lo que hace que consideren el tiempo de práctica como demasiado corto para sus necesidades de aprendizaje, lo cual es retroalimentado desde el diario de campo por el docente. Incluso, se podría considerar que el tiempo de práctica es vital y por ende debería brindarse el necesario para ejecutar todas las actividades en el nivel de complejidad que se amerite, con el fin de garantizar el aprendizaje de los estudiantes (19).

Otro elemento señalado, tiene que ver con el ambiente de la práctica, en donde se deben sostener diversas actividades administrativas y funciones, algunas con cierta autonomía y otras bajo vigilancia constante, allí los estudiantes enriquecen la experiencia con el seguimiento de protocolos y su correcta interpretación, cuidando que su ejecución no se convierta en una tarea rutinaria y mecánica, la cual va en contra vía de la motivación del estudiante (20). Asimismo, en las diferentes circunstancias en que se desempeñe un estudiante dentro del campo de práctica, se convierte, para el docente, una oportunidad única para reconocer particularidades en contexto y retroalimentar en su debida medida, compartiendo experiencias situaciones vividas, logros, entre otros, retroalimentándose mutuamente (17).

Frente al constructo de autocorrecciones, el diario de campo ha resultado ser una herramienta de gran valor, en la medida que las experiencias influencian el modo particular del aprendizaje y la enseñanza (15), con lo cual cada docente puede usar el diario de campo para estimular el aprendizaje de los estudiantes por medio de su autorregulación. Siendo está guiada por la reflexión y el pensamiento crítico, donde los estudiantes son capaces de observar sus debilidades y errores a fin de mejorar y centrarse en ofrecer un servicio de alta calidad.

Se puede decir entonces que el diario de campo provee la posibilidad del perfeccionamiento profesional, esto desde la identificación de fortalezas y debilidades, a fin de atacarlas con la retroalimentación constante con el docente $(15)$. En otras palabras, convertir la práctica en un espacio de deconstrucción y reconstrucción de la preparación profesional del estudiante de enfermería, basado en sus experiencias personales y aprendizajes previstos por el docente.

Por su parte, el constructo de las reflexiones sobre el cuidado, han denotado el papel a desempeñar por el estudiante en la práctica, esto desde su propia concepción del cuidado. En primera instancia señalando que la responsabilidad es muy grande y el cuidado también es un espacio para aprender. Asimismo, señalan que entrar en la práctica les ha dado más herramientas y posibilidades, esto convirtiendo sus aprendizajes en aplicaciones en contexto, lo que podría ser la consolidación de los saberes en la praxis. Esto último relacionado directamente con la motivación del estudiante (20). Lo anterior se enmarca en el reconocimiento de que el cuidado de una persona despierta en el estudiante de enfermería el 
compromiso de proteger la salud del paciente, con ello un mayor apego y valía a su ejercicio profesional (므).

Es importante reconocer que los estudiantes hacen referencia sobre el cuidado como un espacio donde se han enriquecido de experiencias, las cuales fueron tristes y otras agradables, pero que en últimas fortalecen su desarrollo profesional. Es por esto que se considera la formación en el cuidado como algo que sobrepasa lo técnico y procedimental, para situarse en aspectos éticos humanos, y valores que impregnen a los estudiantes de cualidades que lo hagan sentirse seguros e identificados con la profesión (21). Esto último es posible de visibilizar en el diario de campo, porque el estudiante puede plasmar lo que experimenta al entrar en contacto y relación con un paciente en determinadas condiciones, despertando diversas emociones, que debe ser capaz de plasmar para enriquecer su crecimiento y ser retroalimentado por el profesor (16).

En el marco de los hallazgos hasta aquí discutidos, el diario de campo bien se puede considerar como una estrategia educativa para los estudiantes de práctica. Adicional a los hallazgos de la presente investigación, otros autores han señalado que el diario de campo es positivo al permitir visualizar la retroalimentación formal que realiza el docente, la cual puede ser leída y reflexionada en su domicilio, en pro de las mejoras de enseñanza-aprendizaje (17). Esto desde el punto de vista del seguimiento constante y el feedback. De otro lado el diario de campo constituye una herramienta de doble uso, por un lado, como almacén de la bitácora formativa, y de otro lado como puente de comunicación con el docente (18).

Esto último es necesario para luchar contra la dificultad a veces presente en el uso del diario de campo, la cual tiene que ver con la consolidación de todas las reflexiones personales, donde el practicante se sienta en confianza de plasmar sensaciones, experiencias, pensamientos, sentimientos, gestos y todo tipo de componentes cualitativos que enriquezcan los informes descriptivos de actividades y técnicas empleadas (19). En este sentido, es recomendable que los docentes adquieran una mayor consciencia de la importancia del uso del diario de campo como estrategia educativa, afianzando su correcto uso con la intención de potenciar el proceso de enseñanza-aprendizaje de los estudiantes de enfermería (17).

En la educación en enfermería por el mismo carácter humanista de la profesión se debe trascender del aprendizaje instrumental para favorecer la formación ética y humanística, el diario de campo resulta una herramienta útil, porque contribuye a reorganizar el perfil humanista de las ciencias de la salud hacia una situación interactiva. (22) que lleva al estudiante a trascender de su propia individualidad, hacia la comprensión de las relaciones consigo mismo y con los otros, además que propician en los estudiantes el desarrollo de un aprendizaje consciente, significativo y colaborativo, al promover su autonomía (23). Para el profesor, el uso del diario de campo favorece la reflexión sobre la acción que constituye un aspecto fundamental en las prácticas (24) clínicas, lo que contribuye a la revisión del ejercicio docente y del proceso de aprendizaje en forma integral. 


\section{CONCLUSIONES}

El diario de campo es una herramienta que permite la construcción de eticidad por que dota al estudiante y al docente de elementos de aprendizaje significativo favoreciendo abordar la práctica de enfermería desde un abordaje crítico y reflexivo.

La elaboración del diario de campo por parte de los estudiantes identifico algunas dificultades en el aprendizaje, pero permite visibilizar resultados de aprendizaje y elementos actitudinales que contribuyen a la formación integral del futuro profesional como enfermero(a) y como ciudadano.

Los estudiantes requieren de tiempo para su diligenciamiento dentro del mismo escenario práctico.

El diario de campo apoya la detección de conocimientos y habilidades con dificultades y fortalece los que se han adquirido en otros componentes de su carrera profesional.

El pensamiento crítico constituye un elemento esencial en la superación de dificultades y contradicciones presentes en los procesos de aprendizaje.

Fondos: el trabajo fue realizado con el apoyo de la Facultad de Ciencias de la Salud de la Universidad del Tolima.

Agradecimientos: A la universidad del Tolima y estudiantes de la asignatura de enfermería del adulto por el apoyo y colaboración en la realización de este trabajo.

Contribución de los autores: Conceptualización: CPBI, MCOF, CVBS; Conservación de datos: CPBI, MCOF, CVBS Análisis formal: CPBI, MCOF, CVBS; Investigación: CPBI, MCOF, CVBS; Metodología: CPBI, MCOF, CVBS; Visualización: CPBI, MCOF, CVBS; Redacción borrador original: CPBI, MCOF, CVBS; Redacción revisión y edición: CPBI, MCOF, CVBS; Supervisión: $\mathrm{CPBI}$; Administración de proyectos: $\mathrm{CPBI}$; Recursos: $\mathrm{CPBI}$; Todos los autores han leído y aceptado la versión publicada del manuscrito.

Conflictos de intereses: Los autores declaran no tener ningún conflicto de intereses.

\section{REFERENCIAS}

1. Gutiérrez Puertas L. Influencias en el aprendizaje del estudiante en sus prácticas clínicas. Índex de Enfermería. 2016 jul/sept 25(3), 133-134. http://scielo.isciii.es/scielo.php?script=sci arttext\&pid=S1132-12962016000200001

2. Gutiérrez Sabiniana SR. El diario de prácticas clínicas como herramienta de evaluación de los procesos de enseñanza aprendizaje de los alumnos de enfermería (Doctoral dissertation, Universitat d'Alacant-Universidad de Alicante). [Internet]. 2016. https://rua.ua.es/dspace/bitstream/10045/56090/1/tesis sanrafael gutierrez.pdf

3. Díaz Céspedes P. Entre literatura y antropología: el caso del diario de campo de Ernst Jünger en la primera guerra mundial. Desbordes - Revista de Investigaciones de la 
Escuela de Ciencias Sociales, Artes y Humanidades - UNAD/Volumen 8 No. 1. [Internet]. enhttps://hemeroteca.unad.edu.co/index.php/desbordes/article/view/2996/3058

4. Escobar-Castellanos B., Sanhueza-Alvarado O. Patrones de conocimiento de Carper y expresión en el cuidado de enfermería. Enfermería: Cuidados Humanizados, 2018,7(1), 2742. DOI: $10.22235 /$ ech.v7i1.1540

5. López-Cruz V., Hernández-Castañón MA., Mendoza-Zamora A., Villarreal-Ríos E., Gasca-Ramírez MA. Apoyo a estudiantes de enfermería en su primera práctica clínica ante situaciones de estrés. Revista de Enfermería del Instituto Mexicano del Seguro Social, 24(3), 165-170. $\quad$ [Internet]. https://www.medigraphic.com/pdfs/enfermeriaimss/eim-2016/eim163c.pdf

6. Diekelmann N. Curriculum revolution: a theoretical and philosophical mandate for change. En: National League for Nursing, editor. Curriculum revolution: mandate for change. New York, NY (US): p. 137,$57 ; 1988$.

7. Ramírez Perdomo CA. Fenomenología hermenéutica y sus implicaciones en enfermería. Índex de Enfermería, 25(1-2), 82-85; 2016. http://scielo.isciii.es/scielo.php?script=sci abstract\&pid=S1132-12962016000100019

8. Santos Quintero Ml., Garay Núñez JR., Félix Amézquita A., Beltrán Montenegro MDC. EI quirófano como ambiente de aprendizaje en actividades quirúrgicas de la práctica del adulto. Enfermería: Marcando el rumbo de la salud, 77; 2019. https://repositorio.unal.edu.co/handle/unal/54428

9. Sánchez Bracho, M., Fernández, M., Díaz, J. Técnicas instrumentos de recolección de información análisis y procesamiento realizado por el investigador cualitativo. Revista Científica UISRAEL, 8(1), 107.121. [Internet]. 2021. DOI: 10.35290/rcui.v8n1.2021.400

10. Díaz Herrera C. Investigación cualitativa y análisis de contenido temático. Orientación intelectual de revista Universum en Revista General de información y documentación 28 (1), 119-142; 2018. DOI: 10.5209/RGID.60813

11. Tinto Arandes, J.A. El análisis de contenido como herramienta de utilidad para la realización de un investigación descriptiva. Un ejemplo de aplicación práctica utilizando para conocer las investigaciones realizadas sobre la imagen de marca de España y el efecto país de origen provincia. Universidad de los Andes. Mérida Venezuela, (29), 135-173. [Internet]. 2013. https://www.redalyc.org/pdf/555/55530465007.pdf

12. Prácticas de Psicología. Guía para la elaboración del Diario de Campo. Facultad de Ciencias Sociales y Humanas. Universidad de Zaragoza. https://fcsh.unizar.es/sites/fcsh.unizar.es/files/archivos/Psicologia/PracticumPsi/psi pra guia diario campo.pdf

13. Pico Merchán ME. Tobón Correa O. Sentido del rol de profesional de enfermería. Index de Enfermería, 14(51); [Internet]. 2005. http://scielo.isciii.es/scielo.php?script=sci arttext\&pid=S1132-12962005000300004

14. Sánchez Úbeda L. El liderazgo transformacional en enfermería. Universidad de Almería, 2018. http://repositorio.ual.es/bitstream/handle/10835/7828/TFG SANCHEZ\%20UBEDA,\%20LORE NA.pdf? sequence $=1$

15. Souza D., Schubert-Backes V., Delacanal-Lazzari D. Posibilidades en la educación en enfermería: errar Enfermería Universitaria, 14(2), 79-87. [Internet]. 2017.DOl: 10.1016/j.reu.2017.02.005

16. Valderrama Sanabria ML., Peña Pita AP., Clavijo Alvarez LM. Narrativa: el estudiante de enfermería aprendiendo el arte de cuidar. Revista CuidArte, 8(1), 1488-1498. [Internet]. 2014. https://revistacuidarte.udes.edu.co/index.php/cuidarte/article/view/362/756

17. Iturra Briceño L., Riquelme Plaza I. Mejora de la retroalimentación a través del diario de campo en educación superior técnica en el área Salud. Revista Gestión de la Innovación en Educación 
$\begin{array}{lllll}\text { Superior. } & \text { REGIES, } & 2, & 141-162 . & \text { [Internet]. }\end{array}$ http://200.91.9.229/index.php/regies/article/view/48/9

18. Solano M. Noreña A. Gabán A. Gil M. Martínez A. Núñez M. Conca M. Percepción de los alumnos de enfermería de las unidades especiales a través de sus diarios. XII Jornadas de Redes de Investigación en Docencia Universitaria. El reconocimiento docente: innovar e investigar con criterios de calidad. 2014.

19. Siles Gonzalez J., Solano-Ruiz M., Gabán Gutiérrez Á., Gil Estevan MD. Martínez Sabater A., Noreña-Peña A., Conca Martínez M. El diario de prácticas clínicas en la unidad de cuidados intensivos. Investigación y Propuestas Innovadoras de Redes UA para la Mejora Docente, 1665-1676;

2015. https://rua.ua.es/dspace/bitstream/10045/41790/1/2014 XII Jornadas Redes 40.pdf

20. Lopera Betancur MA. Forero Pulido C. Paiva Duque LE. Cuartas VM. El quehacer cotidiano de la enfermera significa soportar la carga. Revista Cuidarte, 7(1), 1262-1270. [Internet]. 2016. https://revistacuidarte.udes.edu.co/index.php/cuidarte/article/view/320/721

21. Godínez Rodriguez M., López Sandoval G. Vivencias de las estudiantes de enfermería durante la práctica clínica. CuidArte "El Arte del Cuidado", 3(6), 58-63. [Internet]. 2014. https://www.google.com/search?q=Vivencias+de+las+estudiantes+de+enfermer\%C3\%ADa+ durante+la+pr\%C3\%A1 ctica+cl\%C3\%ADnica.+CuidArte+\%22El+Arte+del+Cuidado\&rlz=1C1 OKWM esCO928CO929\&oq=Vivencias+de+las+estudiantes+de+enfermer\%C3\%ADa+dura nte+la+pr\%C3\%A1ctica+cl\%C3\%ADnica.+CuidArte+\%22El+Arte+del+Cuidado\&aqs=chrome .69i57.341j0j4\&sourceid=chrome\&ie=UTF-8

22. Trainini JC. Humanización en la práctica médica, Educación Médica, Volumen 21, Issue 2, 2020, pp. 65-66. DOI: 10.1016/i.edumed.2019.12.002

23. Martín-Salinas C. Propuesta de evaluación docente de las prácticas del grado en Enfermería mediante un foro virtual, Educación Médica, 19(6), 348-354. [Internet]. 2018. Disponible en: https://www.sciencedirect.com/science/article/pii/S1575181317301225?via\%3Dihub

24. Bardají Fandos T. El diario reflexivo como herramienta de autoaprendizaje en la formación de enfermería, Nursing (Ed. española), Volume 26, Issue 7. Pages 52-55. [Internet]. 2008. http://www.sciencedirect.com/science/article/pii/S0212538208707702 